\title{
Amputation vs reconstruction in case of catastrophic lower limb
}

\author{
Zamora-Navas $\mathrm{P}^{*}$ \\ Estades-Rubio FJ, Hip Unit, HCU Virgen de la Victoria, Málaga, Spain
}

\begin{abstract}
Catastrophic limb is a challenging situation in which every decision may have dramatic consequences. Every effort to classify or recommend the best treatment has to take into account not only the local circumstances of the injury or the polytrauma context but every component of the personal dimension of the patient, personal, labor, social, etc.

None of the proposed index is able to offer a reliable guide in order to preserve or amputate a mangled limb, not even they are capable to predict the functional final result.

With new reconstructive approaches, surgeons are able to treat injuries which could have been addressed with an amputation in early past. However, the obstinacy in preserving a catastrophic limb may end in a serious disservice to the patient, mainly in terms of personal cost. On the other hand, the insufficiently reasoned decision to cut off a mangled limb may end in an equally undesirable situation with a higher cost for the insurance health service.
\end{abstract}

\section{Introduction}

Mangled limb is defined as one that involves a combination of injuries affecting at least 3 out of the four components of the extremity: vascular, nervous, soft tissues and underlying bone. Basically, it is related to type IIIB and IIIC injuries within the Gustilo and Anderson's classification. However, every work commonly uses criteria that do not always fit within this definition. It is a situation that can lead to amputation in $9 \%$ of the cases in the first 24 hours and in $21 \%$ during the hospitalization [1].

Decision-making becomes more difficult when the patient is within the definition of a polytraumatized one and therefore in a context in which the general situation can influence on local injuries and vice versa by the overload that a limb in such conditions may mean on the instability of the patient and therefore with the interference in the resuscitation manoeuvres.

Furthermore this is not an exclusively technical decision of the surgeon's competence, on the contrary, it should prevail the service provided to the patient where the conservative option for the limb assumes the possibility of submitting the patient to an endless number of surgeries which may result in a painful and minimally functional limb, that hinders rather than ease his professional and personal life. Similarly, a series of items should be taken into consideration, either medicolegal, social or economic factors, that should be part of the decision making process. Moreover, it occurs in an emergency context in which there is not a guideline that satisfactorily helps towards what the best option would be. Unfortunately, this decision making exclusively concerns the features of the injury and obviates any other circumstances [1].

All this becomes particularly dramatic when the age population in which this situation occurs ranges from 20 to 30 years old [2].

The approach to this pathology, in the context of the polytraumatized patient, depends on the initial classification the patient is included in: stable, unstable, in extremis or borderline [3]. Unstable and in extremis situations are those in which the primary amputation has a princeps indication in order to limit the hit that a limb in those conditions, and their possible recovery treatments, produces in the patient's general status. Otherwise, the stable patient demands an effort of reconstruction and awaits for an immediate evolution. After a second look, the surgeon and the patient will decide whether to go along with the initial idea or to reconsider it and then decide on the amputation. In these circumstances, though delayed, the amputation will be still considered as primary. The fourth scenario, the borderline patient, is one in which orthopaedic damage control; lavage, débridement, immobilization of the fracture and revascularization of the interrupted flow. After that, a secondary evaluation will be performed and this may be redirected in one way or another.

The ultimate goal will be to achieve a painless and functional limb, although with some deformity. In any other case, the amputation could be the most adequate decision.

\section{Classification of the open fractures}

The mangled limb is defined as an open fracture with disruption of soft tissues integrity of enough entity that the viability of the limb can be jeopardized. So then, it is necessary to start from a valid classification of the injuries. This will allow the professionals involved set up an initial treatment and finally provide us with a probability of anatomical and functional recovery.

Since 1959, many attempts have been made which eventually have taken shape in the system of Gustillo and Anderson [4,5], which is the

*Correspondence to: Zamora-Navas P, C/ Pintor Sánchez Cotán 7B, 29018 Málaga, Spain, Tel: 0034687066514; E-mail: pzn@uma.es

Received: April 11, 2018; Accepted: April 30, 2018; Published: May 03, 2018 
most widely used one. However, many others have been elaborated, such as the classification of Cauchoix, which have mainly been embraced out in the Anglo-Saxon influence area.

These classifications are designed in order to highlight the contamination and the soft tissue injuries as a risk factor in the fracture evolution.

The Gustillo and Anderson's classification is based on a short series of cases and all of them have been taken from the same anatomical segment, the leg. Being its lack of applicability to fractures of the children and the lesions of the upper limb or pelvis open fractures some of its limitations. Some of the concepts emanated from this classification, such as its prognosis in terms of infection, disorders of the consolidation or prediction of the need for amputation, have been transmitted without testing its correlation in other anatomical segments. Besides, it has been criticized the fact that in this classification elements related to the treatment, such as the possibility of closing the skin with or without graft have been included.

Despite its wide implementation, some other classifications have been proposed. In these, some efforts have been made to include those items considered most important by the orthopedic surgeon, for instance, the development of objective criteria to quantify the bone loss.

One of them is the attempt of the Orthopedic Trauma Association (OTA) [6]. This takes into account five elements: skin injury, muscle injury, bone loss, arterial injury and contamination. So, the skin injure can be divided according to the quantity and quality of skin coverage: skin lacerations that can be approximated, those that are unable to be approximated, and finally the ones associated with extensive dissociation (degloving injuries) (Figure 1). Muscle injury was qualitatively categorized into subgroups according the following: there is no muscle damage in the area, there is a loss, but the muscle remains functional, and finally the injuries with dead muscle and loss of muscle function. Arterial injury is divided into: no injury, artery injury without ischemia and finally artery injury with distal ischemia. On the other hand, the magnitude of contamination is classified as none or minimum -easily removed not embedded in bone or deep soft tissuesand the third grade would be the imbedded in bone or deep soft tissues or with high risk of environmental conditions (barnyard, faecal, dirty water etc.). Finally, bone loss is categorized into: none, bone missing or devascularized but still with some contact among proximal and distal fragment and lastly, segmental bone loss.

\section{Criteria for amputation}

With the acquisition of new skills -such as microsurgical techniques- and the integration of treatment, with guides of the sequence and timing in treating bone, vascular and cutaneous parts, primary amputation is saved for exceptional situations given by the general context of the patient. In any other cases, reconstruction is targeted.

In these circumstances, primary amputation is considered only when limbs are barely attached to the rest of the body by a minimum bond of soft tissues.

A catastrophic limb in the context of a critically-ill patient, a crushed limb with arterial injury and more than 6 hours of warm ischemia and a traumatic amputation with a significant contamination have been recognized as indicative for initial amputation $[7,8]$. On the other hand, there are relative amputation criteria; a significant bone and soft tissue loss, lesion of the posterior tibiae nerve, those cases in which it is accompanied by an ipsilateral injury of the foot and those cases in which it is predicted a very long procedure for the approximation of the soft tissues and bone reconstruction [9]. (Figure 2)

\section{Help to the amputation decision}

There are some factors to be taken into account before amputation. Namely, those related to the variables that affect the injury and the ability of the surgeon to manage them properly, but it should also be considered those regarding the personal and social spheres of the patient and the socioeconomic impact that this decision will have.

There are elements that characterize the lesion and influence the decision making, such as age, general situation of the patient (presence or absence of shock, etc.), time of ischemia from the injury, bone injury, muscle injury, skin injury, venous injury, the amount of contamination and the time in which the treatment is done.

One of the most considered elements is the neurological injury. The absence of sensitivity in the foot's sole has been considered a poor prognostic factor to get a functional recovery. However, a nerve contusion may be expressed as a neurological deficit that does not straightforward correlates with an unrecoverable injury. In such a case, it could be recommended amputation instead of reconstruction that could be successful. In some scales, the presence of an initial neurological injury greatly influences the decision of amputation, that may motivate that the sensitivity and specificity of these scales are not very useful in the clinical practise [10].
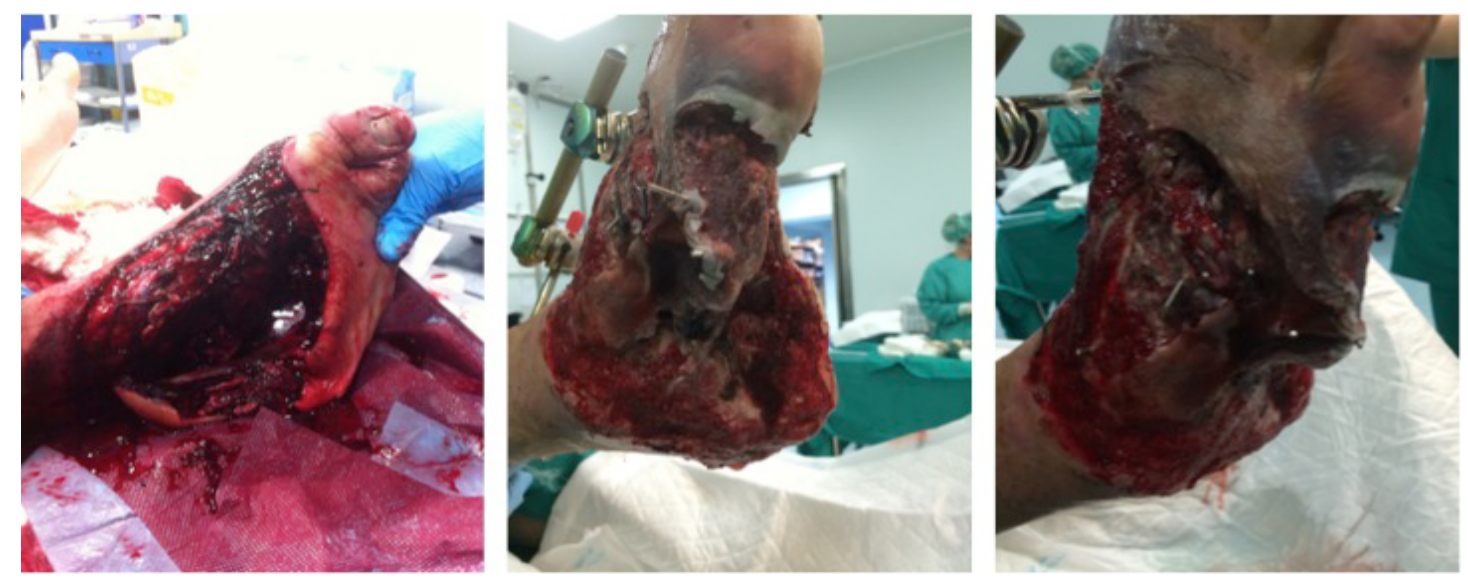

Figure 1. Degloving injuries shadow the final result. After an initial attempt to reconstruct this catastrophic foot, a secondary amputation was considered 


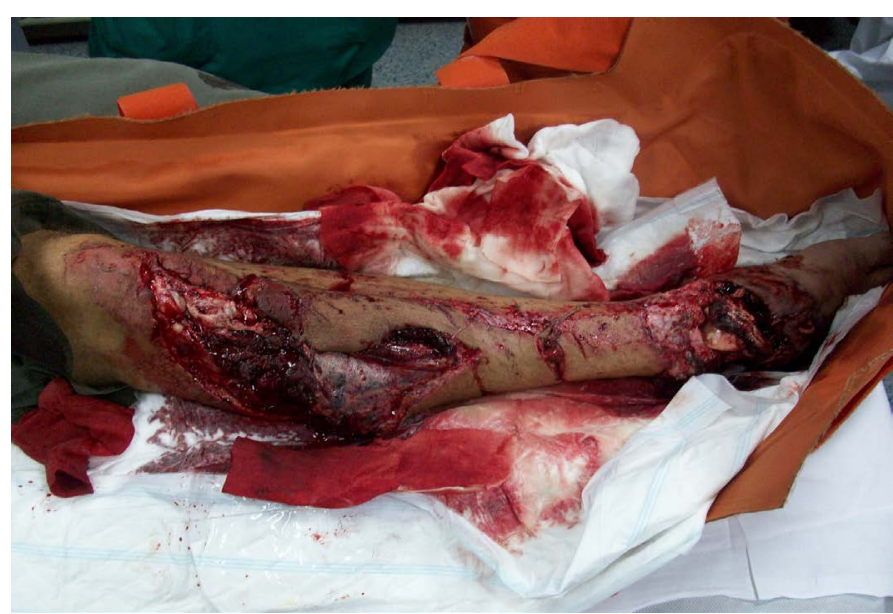

Figure 2. Open fractures of the lower limb associated with a catastrophic foot is often considered an indication for primary amputation

Ideally, these items should be quantified and integrate in a scale. Being so, once a cut-off point is reached, it should allow us to get a treatment recommendation. These scales should be friendly to use, endorsed by an accuracy of $100 \%$ with sensitivity and a specificity of $100 \%$. However, the most widely used guides, MESS, LSI, PSI, NISSSA, HFS-97 do not reach an agreement for the items of judgment to be included. So, we may observe that the load of contamination of the wound is only taken into account on the NISSSA scales and HFS-97. That is also the case of the shock, it is not included in the LSI or PSI scale and, on the other hand, the observation of neurologic injury is included. In the case of the MESS scale, the span of time from the moment of the injury and the beginning of the treatment is not taken into consideration.

In this way, when these scales are submitted to know its power of discrimination, they exhibit a good specificity (between the 0.85 for PSI and HFS-97 and 95\% the LSI), therefore they are able to discriminate the lesions that must be treated with reconstruction, but, on the contrary, they have poor sensitivity (between 0.56 the PSI and 0.67 HFS-97, LSI, MESS and NISSSA), therefore, they have a significant number of failed attempts of reconstructions which eventually end up in secondary amputations being aware that these are accompanied by a mortality approaching $20 \%[7,10,11]$.

Other proposals have been published. These restrict their application to those injuries which would be included in open fractures Gustilo type IIB and, it means without accompanying vascular injury, although they include some important aspects from the general patient's point of view. However, its capacity for recommendation is as limited as those previously mentioned [12].

Despite the attempts made in this area, the efforts have been unsuccessful. The achieved scales have not been able to draw conclusions about a decision, mainly because the available evidence comes from odd quality publications and it has been obtained from both, civilian and military populations -with different cultural backgrounds-. It is still even more difficult to get these recommendations when the purpose is to evaluate a catastrophic upper extremity or in children [13].

Even so, it is possible to reach consensus on the indications of primary amputations in the following cases: when the limb is an uncontrollable source of bleeding that put at risk the patient's life; when a limb is salvageable, but the efforts of reconstruction pose an unacceptable risk to patient's life; when the surgeon considers the limb is non-reconstructable after the joint evaluation of both the orthopaedic and the plastic surgeon [10].

\section{Results}

The evaluation of the outcomes is the true cornerstone of the practical assessment. In these circumstances, to know the patient independent variables influencing the final result will help us to decide the best initial treatment for each one. Both, physical and psychological points of view should be taken in mind.

Its measurement from the perspective of the patient should take into account both; the functional impact -which is usually determined by the type of activity previously developed- and the psychological impact -where body mutilation supposes an important fact but not the only one-. By the same token, social lifestyle and sick leave should not be forgotten. These couldn't be otherwise with repeated attempts of reconstruction with prolonged hospital admissions and cessation of the economic capacity.

There are not so many series that consider the results of one or another decision. To compere, it is necessary to assess its methodological quality. In fact, they include non-homogeneous series, with different number of included patients and with a different followup. The heterogeneity in the selection criteria, in the age of the patients or in the follow-up, makes really difficult to integrate them to conclude the impact on the mental or physical health of these patients. In the same way, the results may be considered scale rating dependent.

The comparison of patients treated with amputation against the reference population supposes to assume an increased morbidity, a lower health profile, a diminished physical function and a worse situation in terms of bodily pain $[14,15]$.

Bosse et al. [16] evaluated the outcomes in terms of both the physical and psychological impacts on the patients subjected to reconstruction and amputation of a catastrophic limb. They were followed-up at 12 and 24 months and 7 years later [17]. The conclusion was that the results measured by the Sickness Impact Profile (SIP) -a scale from 0 to 100; where lower values mean better results and those above 10 pose a severe disability-, are equally bad for both groups and there is no difference between them, apart from the observation that they will be deteriorated along the time.

In the initial work of Bosse and MacKenzie [16], every single assessed dimension in the SIP was worse compared with the reference population, although the dimensions related to psychosocial area -especially communication- approached to normal values (4.1 for the group of patients treated with reconstruction versus 4.0 for those treated by amputation). Although minimally, every value improved at the end of 24 months. It means that only about the $50 \%$ of the patients in both groups were able to return to work. Referring to those who did it, only $58 \%$ of the patients were able to go back to their previous job after 7 years. And those who did so were limited to carry out the previous functions during the $20-25 \%$ of the working time.

In any case, as the authors themselves recognize, none of the aspects of the injury appears to weigh in the functional outcome. This may be due to a selection bias as all the patients under study had similar injuries. On the contrary, the factors that seem to be related to the final result are the characteristics of the patient before the injury. In fact, it seems to be associated with worse functional outcomes having a low level of instruction, belonging to non-white race, lacking private health insurance, smoking or being immersed in the processes of economic dispute. 
However, in other comparative series, the authors found a lower result in physical function for those patients who were submitted to amputation, but similar situation of mental profile and pain control [18]. Georgiadis [19] found a situation of greater disability when the patient is subjected to the reconstruction of the limb. Other authors think that primary amputation implies an overload on physical morbidity, higher than that of patients who are chosen for the reconstruction [20].

In this way, Akula and Col [21] concluded in their meta-analysis that whatever evaluation scale was used, from the patient's point of view, the physical assessment is similar; 39,76 vs. 38.5 for patients who have been treated by amputation with respect to those treated with reconstruction in the SF-36 scale (with a maximum of 100; where 100 is the best imaginable status) and 16.2 vs 13.3 respectively in the SIP scale (scale from 0 to 100; where obtaining a higher score reflects a worse status of health). Meanwhile, in the assessment of the psychological impact, the results are 52.05 vs. 50.7 for the SF-36 scale and 15.6 vs 11.5 in the SIP scale. Finally, the conclusion is that while there is not statistically significant difference in the physical impact, concerning the mental aspect, the result is more favourable in patients who received treatment with reconstruction techniques.

Although catastrophic limb rating scales were designed aiming to help to make decisions about amputation or reconstruction, a second application might be their ability to predict functional outcomes. The comparison performed by Ly et al [22] has tried to establish this relationship. To do this, the population of patients submitted to amputation obtains in the SIP scale an average value for the physical dimension of 17 (very limited), which varies up to 9.5 at 24 months (in the limit of very limited) being the values of psychosocial dimension 12.1 and 9.9 at 6 and 24 months respectively. And these values are obtained from the averages of the MESS scale of 4.4 (the cut-off is set in $\geq 7$ ); LSI of 2.8 (for a cut-off $\geq 6$ ); PSI of 5.6 (with a cut-off $\geq 8$ ); NISSSA of 7.2 (by setting the cut-off $\geq 11$ ) and HFS- 98 of 5.3 (with a cut-off $\geq 11$ ). Besides, it was independent of the punctuation of the different scales; whether they were over its cut-off point or not.

Despite the scales used to assess the outcome, from the patient point of view, it is necessary to know their satisfaction degree about the result of the treatment imposed. Satisfaction is a difficult concept to define, as it is to know what factors govern it, although it seems to be related to physical function. Namely, pain presence or absence, depression symptoms and the ability to return to work after two years of the process. These factors build up the $35 \%$ of the total variability in the satisfaction of the patients [23].

However, due to the accumulation of works that fail to establish a clear difference between the final results of both surgical options, the trend in practice is an attempt of reconstruction except in extreme situations. This decision is motivated by the introduction of new techniques with the aim of decreasing the complications and the recovery time.

The decision of taking either a surgical option or another is far from being solved and needs to incorporate new techniques for the evaluation, taking into account both, the patient and surgeon's, points of view. In addition, it should comprise not only quantitative techniques of analysis, which answers the question "what", but integrate a qualitative analysis that includes "how" and "why", too [24]. Moreover, it should include the patient's viewpoint about abstract measures that professionals have included in their assessment of the results, such as the gain in years with quality of life.
In this regard, the surgeon, in the purpose of a better result and supported by the introduction of new reconstruction techniques, maintains their preference in reconstructive techniques. In any case, the professional perception of the gain in years with quality of life comparing amputation vs reconstruction is low. However, the loss in quality of life compared with the reference population is around 4 QALYS, while in other fractures it is better considered, for instance 0.18 QALYS for vertebral fractures and 0.16 for hip fractures. On the other hand, the patient perceives a much higher loss of quality of life, 6.21, with an important difference if they have been subjected to techniques of reconstruction or amputation although they have suffered different complications. So, the loss of quality of life per year assigned by the surgeon is estimated at 0.97 and 0.95 for reconstructions vs amputations, while the patient considers a loss of 0.83 and 0.67 respectively [25].

\section{Complications}

Whatever the therapeutic option is, the treatment of a mangled limb supposes not only the need of a number of surgeries, but also that it will certainly present a major number of complications and even mortality. Not to forget the increase in the cost of the treatment.

Among the options, reconstruction is the one that is accompanied of a greater number of necessary procedures to achieve the final result. In the same way, this requires a longer period of hospitalization as well as a higher occurrence of complications like osteomyelitis and even the need of secondary amputations [26].

Those patients in whom reconstruction has been attempted and eventually suffer a secondary amputation present more complications, doubling in percentage, cases of wound infection respect to those whose reconstruction was successful ( $68 \%$ vs. $23 \%)$ and osteomyelitis (40 vs. $8.6 \%$ ) compared with primary amputation group. The same must be expected related to disorders of consolidation ( $48 \%$ vs. $31 \%$, respectively), equalizing the proportions of patients with impaired articulation (12 vs. 9.4\%) in both groups. Moreover, the group of secondary amputations doubled ( 24 vs. $10.7 \%$ ) the complications in the stump when it is compared with patients who received an amputation as the first procedure after the trauma.

\section{Economic valuation}

Whatever the decision is, reconstruction or amputation, it must be taken into account that this is a process with bulk economic cost and serious social implications. Thus, the reconstructive option involves long-term treatment with several visits to the operating theatre, which varies from 4-7 [19].

The allocation of costs should include both, the initial process as the subsequent readmissions, medical and therapist visits and investment in prosthesis equipment. Although the time of hospitalization of the first episode is similar (17 days), the difference happens in the need of readmission that consumes up to an average of 60.7 days for the group of patients who get reconstructive treatment instead of 35.4 of those treated by amputation. For the rest of the resources consumption in terms of visits to the surgeon (13.9 vs. 15) or occupational therapist (34.2 vs. 38.2) both group are similar [27].

Being important the difference in cost in those sections, what consumes more resources is the issue of the orthotic implants. These must be changed once per year during the first two years and once every 5 years since then and for the rest of their lives. In the most conservative estimations the costs overhead to $\$ 509,275$ compared with $\$ 163,282$ [27]. 
So, although initially the amputation surgery cost is lower than the reconstruction one, at the end of the first two years of treatment, the cost comes to the same amount (average $\$ 84316$ in the case of the reconstruction vs $\$ 91106$ for amputations). Thereafter, the spending devoted to maintenance of the walking aids in amputated patients multiplies economic investment with regard to reconstructions27. It is estimated that, once included the cost of repair and reposition of the orthotics, for an expected average of 40 years, the difference in $\$$ of 2002 would be between $\$ 93606$ and $\$ 154636$. This difference may be bigger the younger the patient is.

\section{Conclusion}

In the treatment of a catastrophic limb in the context of a polytraumatized patient, a primary amputation should be reserved for those patients classified as in extremis or unstable status. Secondary amputation is conditioned by either the occurrence of complications such as infection or considering the final functionality obtained.

None of the two options in the treatment of catastrophic limbs concludes in a satisfactory result. Any of both therapeutic alternatives involves a physical and emotional wear that could be disastrous for the patient.

The financial economic investment is higher in cases of amputation. The introduction of microsurgical reconstruction techniques has led to an improvement in the assistance allowing a greater number of salvations, but our target is to provide the patient with "the best treatment" instead of the "technically-possible" for the surgeon.

The lack of consideration of the patient dependent factors may lead to the situation defined by Tornetta of a patient divorced, unemployed, disabled, and depressed [28].

\section{References}

1. de Mestral C, Sharma S, Haas B, Gomez D, Nathens AB (2013) A contemporary analysis of the management of the mangled lower extremity. $J$ Trauma Acute Care Surg 74: 597-603.

2. Sabapathy SR (2006) Management of complex tissue injuries and replantation across the world. Injury 37: 1057-1060.

3. Pape HC, Giannoudis PV, Krettek C, Trentz O (2005) Timing of fixation of major fractures in blunt polytrauma: role of conventional indicators in clinical decision making. J Orthop Trauma 19: 551-562.

4. Gustilo RB, Anderson JT (1976) Prevention of infection in the treatment of one thousand and twenty-five open fractures of long bones: retrospective and prospective analyses. J Bone Joint Surg Am 58: 453-458.

5. Gustilo RB, Merkow RL, Templeman D (1990) The management of open fractures. $J$ Bone Joint Surg Am 72: 299-304. [Crossref]

6. A new classification scheme for open fractures (2010) Orthopaedic Trauma Association: Open Fracture Study Group. J Orthop Trauma 24: 457-64.

7. Hansen ST Jr (1987) The type-IIIC tibial fracture. Salvage or amputation. J Bone Joint Surg Am 69: 799-800. [Crossref]

8. Lange RH, Bach AW, Hansen ST Jr, Johansen KH (1985) Open tibial fractures with associated vascular injuries prognosis for limb salvage. J Trauma 25: 203-208.

9. Johansen K, Daines M, Howey T, Helfet D, Hansen ST Jr (1990) Objective criteria accurately predict amputation following lower extremity trauma. J Trauma 30: 568-72.

10. Hoogendoorn JM, van der Werken C (2002) The Mangled Leg Decision-Making Based on Scoring Systems and Outcome Decision-Making Based on Scoring Systems and Outcome Review Article. Eur J Trauma Emerg Surg 28: 1-10.

11. Higgins TF, Klatt JB, Beals TC (2010) Lower Extremity Assessment Project (LEAP)-the best available evidence on limb-threatening lower extremity trauma. Review. Orthop Clin North Am 41: 233-9.
12. Rajasekaran S, Sabapathy SR, Dheenadhayalan J, Sundararajan SR, Venkatramani H, et al. (2015) Ganga hospital open injury score in management of open injuries. Eur $J$ Trauma Emerg Surg 41: 3-15.

13. Fractures (Complex): Assessment and Management. Editors National Clinical Guideline Centre (UK) (2016) Source London: National Institute for Health and Care Excellence (UK) Feb? National Institute for Health and Care Excellence: Clinical Guidelines.

14. Gunawardena NS, Seneviratne Rde A, Athauda T (2006) Functional outcomes of unilateral lower limb amputee soldiers in two districts of Sri Lanka. Mil Med 171: 283-7.

15. Smith DG, Horn P, Malchow D, Boone A, Reiber GE, et al. (1995) Prosthetic history, prosthetic charges, and functional outcome of the isolated, traumatic below-knee amputee. J Trauma 38: 44-47. [Crossref]

16. Bosse MJ, MacKenzie EJ, Kellam JF, Burgess AR, Webb LX, et al. (2002) An analysis of outcomes of reconstruction or amputation after leg-threatening injuries. $N$ Engl J Med 347: 1924-1931. [Crossref]

17. MacKenzie EJ, Bosse MJ, Kellam JF, Pollak AN, Webb LX, et al. (2006) Early predictors of long-term work disability after major limb trauma. $J$ Trauma 61: 688-94.

18. Dagum AB, Best AK, Schemitsch EH, Mahoney JL, Mahomed MN, et al. (1999) Salvage after severe lower-extremity trauma: are the outcomes worth the means? Plast Reconstr Surg 103: 1212-20.

19. Georgiadis GM, Behrens FF, Joyce MJ, Earle AS, Simmons AL (1993) Open tibial fractures with severe soft-tissue loss. Limb salvage compared with below-the-knee amputation. J Bone Joint Surg Am 75: 1431-41.

20. Seekamp A, Regel G, Ruffert S, Ziegler M, Tscherne H (1998) Amputation or reconstruction of IIIB and IIIC open tibial fracture. Decision criteria in the acute phase and late functional outcome. Unfallchirurg 101: 360-369. [Crossref]

21. Akula M, Gella S, Shaw CJ, McShane P, Mohsen AM (2011) A meta-analysis of amputation versus limb salvage in mangled lower limb injuries--the patient perspective. Injury 42: 1194-7.

22. Ly TV, Travison TG, Castillo RC, Bosse MJ, MacKenzie EJ (2008) LEAP Study Group. Ability of lower-extremity injury severity scores to predict functional outcome after limb salvage. J Bone Joint Surg Am 90: 1738-43.

23. O’Toole RV, Castillo RC, Pollak AN, MacKenzie EJ, Bosse MJ (2008) LEAP Study Group. Determinants of patient satisfaction after severe lower-extremity injuries. $J$ Bone Joint Surg Am 90: 1206-11.

24. Aravind M, Shauver MJ, Chung KC (2010) A qualitative analysis of the decisionmaking process for patients with severe lower leg trauma. Plast Reconstr Surg 126: 2019-29.

25. Chung KC, Shauver MJ, Saddawi-Konefka D, Haase SC (2011) A decision analysis of amputation versus reconstruction for severe open tibial fracture from the physician and patient perspectives. Ann Plast Surg 66: 185-91.

26. J Harris AM, Althausen PL, Kellam J, Bosse MJ, Castillo R (2009) Lower Extremity Assessment Project (LEAP) Study Group. Complications following limb-threatening lower extremity trauma. J Orthop Trauma 23: 1-6.

27. MacKenzie EJ, Jones AS, Bosse MJ, Castillo RC, Pollak AN, et al. (2007) Health-care costs associated with amputation or reconstruction of a limb-threatening injury. J Bone Joint Surg Am 89: 1685-1692. [Crossref]

28. Tornetta P 3rd, Olson SA (1997) Amputation versus limb salvage. Instr Course Lect 46: 511-518. [Crossref]

Copyright: (C2018 Zamora-Navas P. This is an open-access article distributed under the terms of the Creative Commons Attribution License, which permits unrestricted use, distribution, and reproduction in any medium, provided the original author and source are credited. 